\title{
Dexamethasone and oxygen therapy in care home residents with diabetes: a management guide and algorithm for treatment: a rapid response action statement from the European Diabetes Working Party for Older People (EDWPOP) and European Geriatric Medicine Society (EuGMS)
}

\author{
Alan James Sinclair ${ }^{1,2} \cdot$ Stefania Maggi ${ }^{3,4} \cdot$ Ahmed Hassan Abdelhafiz ${ }^{2,5} \cdot$ Nicola Veronese $^{4,6}$. \\ Leocadio Rodriguez-Manas ${ }^{2,7} \cdot$ Isabelle Bourdel-Marchasson ${ }^{4,8}$
}

Received: 10 February 2021 / Accepted: 18 February 2021 / Published online: 15 April 2021

(c) The Author(s) 2021

\begin{abstract}
This statement addresses the need to provide clinically relevant and practical guidance for long-term care staff working in care homes and other stakeholders engaged in the care of residents who require consideration for dexamethasone and oxygen therapy. It had been provided following a series of consensus discussions between the EDWPOP and the EuGMS in January and February 2021. Its main aim is to minimise morbidity and mortality from serious acute illnesses including COVID-19 requiring these treatments within the long-term care sector.
\end{abstract}

Keywords Long-term care · Diabetes · Oxygen · Dexamethasone $\cdot$ COVID-19

This guidance was prepared by two European medical organisations with multidisciplinary input to:

- Alert long-term care facilities (LTC, residential care homes including nursing homes) that they may be expected to manage residents with diabetes with hypoxaemia and pneumonia who require oxygen therapy and/ or dexamethasone

Alan James Sinclair

alan.sinclair@kcl.ac.uk

fDROP and King's College, London, UK

2 European Diabetes Working Party for Older People (EDWPOP), London, UK

3 CNR-IN (Aging Branch), Padua, Italy

4 European Geriatric Medicine Society (EuGMS), Vienna, Austria

5 Rotherham General Hospital, Rotherham, UK

6 University of Palermo, Palermo, Italy

7 University Hospital of Getafe, Madrid, Spain

8 Chu-Bordeaux, Bordeaux, France
- Minimise morbidity and mortality from serious acute illnesses including COVID-19 which require these treatments

- Emphasise that those at the highest risk of poor outcomes from serious acute illness in long-term care are those who have: frailty, several existing medical conditions, such as cardiovascular disease or respiratory disease, diabetes complications, treatment with steroids, a life expectancy $<6$ months

- Emphasise that residents requiring oxygen therapy and/or dexamethasone can deteriorate quickly — this is a medical emergency situation

This guidance is addressed to: managers/senior nursing staff of long-term care (LTC) facilities, other care staff, community nursing and diabetes staff, primary care providers including general practitioners.

\section{Background}

The significant impact of COVID-19 on the care home or LTC population has been dramatic in terms of increased morbidity and mortality. In many countries, LTC has been the epicentre of the pandemic, with the incidence and 
severity of illness and mortality shifted towards older people particularly those with multiple comorbidities, such as diabetes, hypertension, and cardiovascular disease [1]. In addition, a quarter of residents have diabetes and two-thirds may have frailty which is a better predictor of intensive care unit (ICU) outcomes than age or other factors [2].

Residents in LTC are a highly co-morbid population who are particularly vulnerable to developing rapid serious acute illness as has been demonstrated with COVID-19 infection. The huge burden on hospital-based care for managing COVID-19 has meant that many residents with this infection remain in LTC and are not referred to hospital, with care staff being expected to take on additional care not previously considered to be within their remit. This includes administering oxygen and/or dexamethasone therapy.

\section{Dexamethasone and oxygen therapy}

Dexamethasone (a corticosteroid) treatment is associated with improved survival and clinical benefit with people with severe COVID-19 [4]. The results show that dexamethasone reduces 28 -day mortality substantially among patients who received oxygen or ventilation at the time of randomisation in the UK Phase 3 open label randomised clinical trial, RECOVERY1 [4]. Dexamethasone has a favourable benefit-risk profile when used in those with severe pneumonia. Short-term use ( $<2$ weeks) is associated with few side effects apart from hyperglycaemia in non-diabetic individuals [5] but may worsen glucose control in those with diabetes mellitus by reducing peripheral use of glucose. It is also important to monitor for fluid and sodium retention.

In LTC, dexamethasone is now also being prescribed when a resident requires oxygen therapy to correct hypoxaemia and maintain satisfactory arterial oxygen saturation (92-96\%), unless there is a background of chronic lung disease when a level of $>88 \%$ may be more realistic. An oxygenation strategy using high-flow nasal cannulae [6] may be another option that could be considered within a wellresourced LTC setting.

The format for this guide on dexamethasone and oxygen therapy has been developed from earlier UK guidance [3] and designed to support clinical decision-making in long-term care facilities. As such, the guidance will need to be interpreted in the light of the availability of skilled personnel, adherence to local/national regulations for oxygen provision within LTC including monitoring of oxygen delivery and blood oxygen saturation (pulse oximetry), monitoring of blood glucose and ketones, fluid administration limits, and overall level of care that is available. Whenever possible, other usual care practices for a resident with diabetes should be maintained.

\section{Implementing this guidance}

The implementation of this European guidance will require it to be locally adapted and agreed between communitybased health professional teams and GP-led services which interact with LTC to provide direct clinical care. Crucially, these treatments require respect of the individual's values and preferences or that of their family if consent not possible.

Communication between all relevant parties (care homes, community services, primary care, local hospitals) may be enhanced by technology for virtual reviews/case conferences to minimise contacts for healthcare professionals [3]. For residents recently discharged from hospital on dexamethasone, this guidance can still be provide additional advice on continuation of this treatment as recommended by local clinicians/specialists.

\section{Infection control and prevention}

Appropriate infection control precautions should be in place including: Isolation facilities where required, as well as personal protective equipment (PPE). Further information is available at: World Health Organisation (WHO) WHO2019-nCoV-IPC_long_term_care-2021.1-eng(1).pdf.

This guidance should only be considered when sufficient support is available to safely administer treatments within the LTC/care home environment. Liaison with a respiratory specialist is advisable. This guidance is not to be employed when it is clear that admission to hospital is necessary to avoid further deterioration in the health status of the resident. 
- Resident with respiratory failure (e.g. COVID-19) requiring oxygen therapy

for continued hypoxaemia

- Oxygen saturation $<92 \%$ (COPD $<88 \%$ by pulse oximeter)

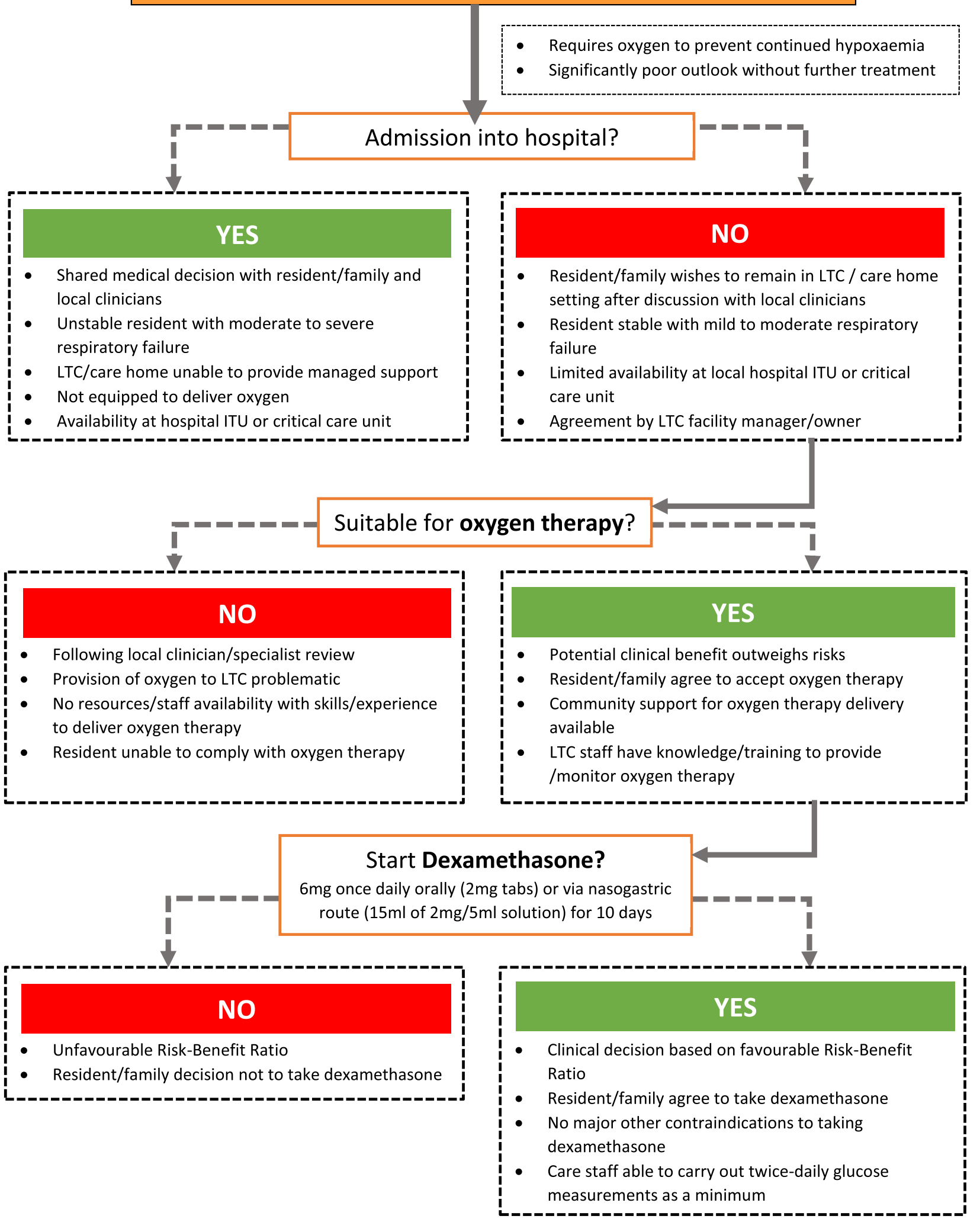

Fig. 1 Algorithm for oxygen and dexamethasone therapy in long-term care (LTC) 


\section{Advice for managers and owners of long-term care facilities}

- In liaison with local clinicians, ensure that there is a clear plan in place for continuing any anti-infective therapy that has been prescribed: this may require supervision and monitoring of intravenous route of administration.

- Equip your care home with sufficient capillary blood glucose strips (with a meter), and strips for ketones if possible.

- Ensure you and your team are equipped, resourced and trained sufficiently to take on oxygen delivery and monitoring.

- Discuss with local clinicians/specialists the severity of the respiratory failure and requirements for oxygen delivery (e.g. cylinders), face masks, and standard/high flow nasal cannulae.

- Ensure your care staff can reliably adjust oxygen flow rates according to oximetry oxygen saturation and the nature of the underlying illness (e.g. Covid-19 or chronic obstructive pulmonary disease - COPD): see Box 1 and algorithm (Figure 1).

- Ensure that your care staff are familiar with administering dexamethasone treatment and its cautions/side-effects: Box 2 and Figure 1.

- Agree with local clinicians what glucose targets and insulin regimen(s) should be adopted for a resident on dexamethasone - see Box 3.

- Engage with your resident/family/carer and local clinician team to discuss thromboprophylaxis (caution with dexamethasone), gastro-protection (caution with dexamethasone), and advance care planning particularly if the resident's health status is not stable.

- Maintain a written record of a resident's blood glucose, respiratory rates, pulse oximetry oxygen levels, oxygen flow rates, medications, temperature, food chart and body weight.

- Ensure good communication with your local diabetes and respiratory specialist nurses (if available), the community nursing service, and with your primary care team who will want to provide you and your staff with support and guidance at all times.

In Box 1, guidance is given on managing oxygen flow rates via nasal cannulae which will require supervision by a trained nurse or other local clinician initially. In Fig. 1, an algorithm has been provided to manage a resident with mild to moderate hypoxaemia requiring oxygen therapy and dexamethasone using a step-by-step approach. We recognise that some LTC settings will face difficulties in providing this level care.
In Box 2, a set of important clinical alerts has been provided which should be seen as warning alerts that should prompt seeking medical advice and even calling the emergency services in some circumstances.

In Box 3, we provide guidance on the management of glucose levels whilst taking dexamethasone, and suggest insulin regimens that should be considered.

\section{Box 1: Oxygen flow rate titration guidance}

- Guidance to be agreed by local clinicians supported by specialist physician

- Start oxygen via nasal cannulae at 1-2 litres per minute and increase at 30 minute intervals to maintain oxygen saturation at $92-96 \%$ (up to 4-6 litres per minute); undertake frequent oximetry readings/clinical observations

- For residents with longstanding COPD (Chronic Obstructive Pulmonary Disease) aim to achieve an oxygen saturation of $88-92 \%$

- In COPD, start 24-28\% oxygen via a Venturi mask or via nasal cannulae at a flow rate at 0.5-1 litre per minute, and increase flow rate at 30-45 minute intervals to a maximum of 2 litres per minute to achieve target oxygen saturation; undertake frequent oximetry readings/clinical observations

- Maintain the oxygen flow rate when target saturation (by oximetry) is achieved and ensure that saturation does rise above or below the target range 


\section{Box 2: clinical alerts}

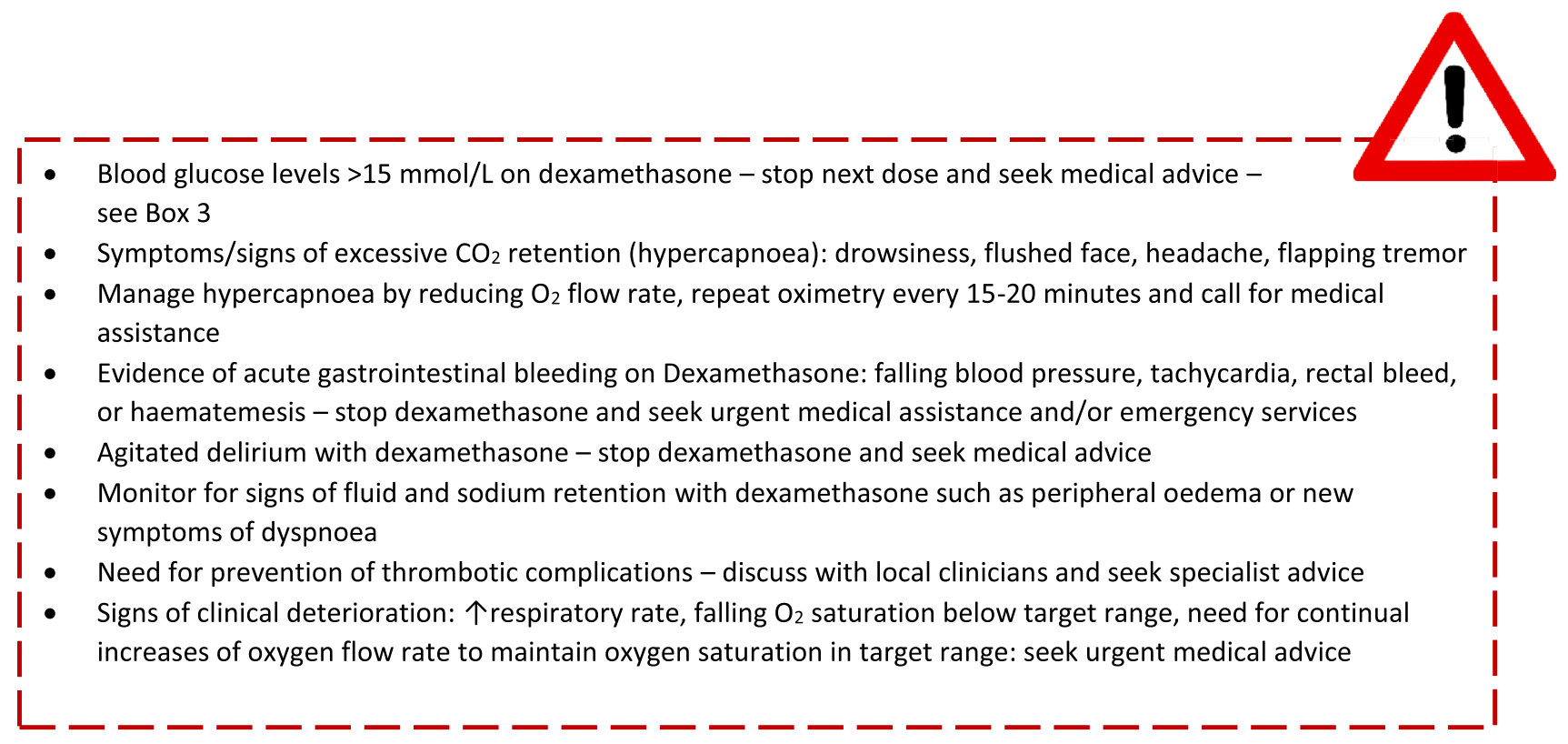

\section{Box 3: Insulin use in residents with diabetes requiring dexamethasone}

- Arrange HbA1c as a measure of pre-dexamethasone glucose control and send off a blood sample for standard haematology and biochemistry including an eGFR

- Aim to check blood glucose levels 8-12 hrly (but 6hrly if resources and/or trained staff available)

- If BG in target (7 to $12 \mathrm{mmol} / \mathrm{L}$ ) continue oral glucose-lowering medication but avoid metformin, SGLT-2 inhibitors, GLP-1 receptor agonists and sulphonylureas during acute illness.

- If $B G$ above target $>12 \mathrm{mmol} / \mathrm{L}$ : exclude diabetic ketoacidosis (DKA) ${ }^{3}$; add short acting insulin (e g Novorapid, Humalog or Apidra) as required 4 units every 6 hrs to bring down BG to target 7 to $12 \mathrm{mmol} / \mathrm{L}$ and continue to monitor.

- If $\geq 2$ consecutive blood glucose readings are $>12 \mathrm{mmol} / \mathrm{L}$ :

A. Patients on oral hypoglycaemic therapy:

- Maintain dose of oral glucose-lowering agent and use short acting insulin 4 units every 6 hrs to bring to target range of 7-12 $\mathrm{mmol} / \mathrm{L}$;

- If target range not achieved, add basal insulin (Lantus, Levemer, Degludec) starting at a dose of 0.15 units $/ \mathrm{kg}$ with gradual titration and continue as required short acting insulin 4 units every $6 \mathrm{hrs}$.

B. Patients already on basal, intermediate acting insulin or basal-bolus regimen:

- Increase basal or intermediate acting insulin by $20 \%$ with gradual titration and continue as required short acting 4 units every 6 hrs. 
Funding No funding was sought or obtained in creating this work.

\section{Compliance with ethical standards}

Conflicts of interest The authors declare no conflicts of interest.

Ethical standards The work needed in creating this document complied with all relevant ethical standards. This work did not involve patient care or clinical trial involvement of patients. Ethical Approval was not required for the creation of this document.

Informed consent The issue of informed consent was not relevant to this work as no patients were directly involved.

Open Access This article is licensed under a Creative Commons Attribution 4.0 International License, which permits use, sharing, adaptation, distribution and reproduction in any medium or format, as long as you give appropriate credit to the original author(s) and the source, provide a link to the Creative Commons licence, and indicate if changes were made. The images or other third party material in this article are included in the article's Creative Commons licence, unless indicated otherwise in a credit line to the material. If material is not included in the article's Creative Commons licence and your intended use is not permitted by statutory regulation or exceeds the permitted use, you will need to obtain permission directly from the copyright holder. To view a copy of this licence, visit http://creativecommons.org/licenses/by/4.0/.

\section{References}

1. World Health Organisation (WHO) (2021) COVID-19 Clinical management: living guidance. Available at: COVID-19 Clinical management: living guidance (who.int)

2. Sinclair AJ, Abdelhafiz AH (2020) Age, frailty and diabetes-triple jeopardy for vulnerability to COVID-19 infection. EClinicalMedicine 23(22):100343

3. Sinclair A, Dhatariya K, Burr O, Nagi D, Higgins K, Hopkins D et al (2020) Guidelines for the management of diabetes in care homes during the Covid-19 pandemic. Diabet Med. https://doi. org/10.1111/dme.14317

4. (2020) Rapid Evaluation of Covid-19 Therapy (RECOVERY trial). University of Oxford. https://www.recoverytrial.net/results

5. World Health Organisation (WHO) (2020) Coronavirus disease (COVID-19): dexamethasone. Available at: Coronavirus disease (COVID-19): Dexamethasone (who.int)

6. National Institutes of Health. USA (2020) Covid-19 Treatment Guidelines. Oxygen and Ventilation. Available at: Oxygenation and Ventilation I COVID-19 Treatment Guidelines (nih.gov)

Publisher's Note Springer Nature remains neutral with regard to jurisdictional claims in published maps and institutional affiliations. 